\title{
Induction du stress oxydatif chez l'homme suite à la bioconcentration des éléments traces métalliques (cadmium et plomb) par voie trophique à Kpémé (Sud du Togo)
}

\author{
Mamatchi MELILA ${ }^{1 *}$, Wiyao POUTOULI ${ }^{3}$, Kou'santa AMOUZOU ${ }^{1}$, \\ Gado TCHANGBEDJI ${ }^{2}$, Maclewe TCHAOU ${ }^{1}$, Ananivi DOH ${ }^{1}$ et Chantal GOTO ${ }^{4}$ \\ ${ }^{1}$ Laboratoire de Biochimie Appliquée à la Nutrition et à l'Alimentation, Département de Biochimie/Nutrition, \\ Faculté des Sciences, Université de Lomé, Togo. \\ ${ }^{2}$ Laboratoire de Gestion, Traitement et Valorisation des Déchets (GTVD), Département de Chimie, \\ Faculté des Sciences, Université de Lomé, Togo. \\ ${ }^{3}$ Laboratoire de Biologie Animale, Faculté des Sciences, Université de Lomé, Togo. \\ ${ }^{4}$ Laboratoire de l'Institut Togolais de Recherche Agronomique (ITRA), Lomé, Togo. \\ *Auteur correspondant, E-mail: mamatchi@yahoo.fr ; Tel: (228)90747178 /(228)98273769.
}

\section{RESUME}

La pollution due aux rejets des déchets phosphatés à Kpémé par l'usine de la SNPT au Togo, provoque la bioaccumulation du $\mathrm{Cd}$ et du $\mathrm{Pb}$ par les produits de mer et agricoles consommés par l'homme qui est ainsi contaminé. Nous avons voulu par cette étude déterminer l'impact de la bioaccumulation du $\mathrm{Cd}$ et du $\mathrm{Pb}$ sur le stress oxydatif dans cette zone, chez l'homme. Les teneurs en métaux lourds toxiques $(\mathrm{Cd}$ et $\mathrm{Pb})$ sont déterminées dans le sang humain à Gbodjomé (zone de référence) et à Kpémé où les populations consomment des aliments contaminés, par spectrophotométrie d'absorption atomique à l'électrothermie; de même que certains biomarqueurs (MDA et FRAP) du stress oxydatif. Dans l'ensemble, les résultats ont montré une peroxydation lipidique et une baisse du potentiel total antioxydant du plasma sanguin à cause de la présence du $\mathrm{Cd}$ et $\mathrm{du} \mathrm{Pb}$, qui provoquent un stress oxydatif dans l'organisme humain qui présente une disponibilité à la bioaccumulation de ces métaux à Kpémé. Les biomarqueurs du stress oxydatif chez l'homme peuvent être donc des indicateurs de l'exposition des sujets aux éléments traces métalliques dans les zones de pollution environnementale.

() 2012 International Formulae Group. All rights reserved.

Mots clés : SNPT, bioaccumulation, sang humain, MDA, FRAP.

\section{INTRODUCTION}

Les éléments traces métalliques constituent le principal facteur du stress oxydatif (Yildirim et al., 2011 ; Mélila et al., 2012) chez les mammifères. Les métaux lourds oxydent en effet les lipides et les protéines et forment des produits de dégradation à forte activité radicalaire qui produisent des espèces réactives de l'oxygène (ROS) (Servais, 2004). Dans le cas d'un bon fonctionnement de l'organisme, les ROS sont des produits essentiels. L'équilibre - avoir des ROS en quantité nécessaire au bon fonctionnement cellulaire et pas en excès pour ne pas trop oxyder - est maintenu au sein de la cellule par des systèmes d'élimination des 
ROS (Aït-Aïssa et al., 2003). Dans la cellule, il existe donc un équilibre entre la production des ROS et leur élimination. Le stress oxydant est classiquement défini comme un déséquilibre en faveur de la production des ROS qui conduit à une oxydation accrue des composants cellulaires essentiels. Ce déséquilibre pro-oxydant/antioxydant peut avoir une origine exogène: molécules oxydantes, toxines telles que les métaux lourds toxiques; ou une origine endogène : disfonctionnements de certaines sources de production et systèmes d'élimination des ROS (Favier, 2003; Servais, 2004). Ainsi, pour neutraliser les effets toxiques des radicaux libres, les mammifères possèdent un système d'antioxydants (Nordberg et Arner, 2001; Almeida et al., 2002 ; Pandey et al., 2003) qui comprend des enzymes antioxydantes et de nombreuses molécules comme la glutathion (GSH), les vitamines A, C et E (Yildirim et al., 2011). Lorsque les défenses antioxydantes sont affaiblies ou dépassées, le stress oxydatif peut provoquer l'inactivation enzymatique et la peroxydation lipidique (Halliwell et Gutteridge, 1989; Farombi et al., 2007 ; Yildirim et al., 2011 ; Mélila et al., 2012). Par ailleurs, la Malondialdéhyde (MDA), utilisée comme marqueur de la peroxydation lipidique reflète le statut oxydatif des individus exposés aux xénobiotiques (Charissou et al., 2004) de même que le potentiel total antioxydant du plasma sanguin (Ferric Reducing Antioxidant Power) «FRAP » ( Legault, 2007 ; Agbonon et Gbéassor, 2009). Il a été démontré d'autre part que la formation de la MDA est un indicateur de la pollution de l'environnement (Farombi et al., 2007; Yildirim et al., 2011 ; Mélila et al., 2012). Au Togo, des études (Bodjok, 2003; Tchangbédji et al., 2003 ; Abbé, 2004) ont montré que la côte togolaise fait l'objet d'une pollution à cause du traitement du minerai de phosphate par l'usine de la Société Nouvelle des Phosphates du Togo (SNPT). Les effluents rejetés par cette usine dans la mer d'une part et les poussières engendrées dans l'atmosphère d'autre part, renferment des traces de métaux lourds tels que le $\mathrm{Cd}$ et le $\mathrm{Pb}$, contaminés à l'homme par voies cutané, respiratoire et trophique à travers la chaîne alimentaire (Abbé, 2004 ; NSE/ONUDI, 2007). Cependant, bien que des études (NSE/ONUDI, 2007) aient montré l'exposition des populations de la côte togolaise aux métaux lourds toxiques, aucune étude scientifique n'a été entreprise pour évaluer le taux de présence de ces métaux dans le sang et l'impact de l'intoxication par la bioaccumulation de ces métaux sur le stress chez l'homme. La présente étude est effectuée afin de déterminer le niveau d'intoxication des hommes par le $\mathrm{Cd}$ et le $\mathrm{Pb}$ et le stress oxydatif engendré par ces métaux. Nous rapportons ainsi donc pour la première fois les teneurs du $\mathrm{Cd}$ et du $\mathrm{Pb}$ dans le sang humain dans la localité de Kpémé, localité polluée par le traitement du phosphate togolais ; mais aussi, l'impact biochimique de ces métaux dans l'organisme à travers le stress oxydatif en se référant au potentiel total antioxydant du plasma sanguin (FRAP) et à la production de la malonedialdéhyde (MDA), un produit de la peroxydation lipidique, qui sont identifiés par des études (Legault, 2007 ; Farombi et al., 2007 ; Agbonon et Gbéassor, 2009 ) comme des indicateurs du stress oxydatif et marqueurs biologiques de la pollution environnementale.

\section{MATERIEL ET METHODES \\ Matériel}

Il s'agit des échantillons de sang prélevés sur des sujets à Kpémé, lieu de pollution et à Gbodjomé site servant de contrôle, situé en amont à environ $11 \mathrm{Km}$ de l'usine de la SNPT et qui ne fait donc pas objet de cette pollution ;

- Des appareils et matériels de laboratoire ;

- Des réactifs de laboratoire tels que : le $\beta$-2-mercaptoétanol, le TritonX100, le peroxyde d'hydrogène, le tampon Tris- $\mathrm{HCl}$, l'acide chlorhydrique $(\mathrm{HCl})$, la solution d'Ethyle Diamine Tétra-Acétique (EDTA), qui sont fournis par LABOSI OULSHY-LECHATEAU-France ; le n-butanol et l'éthanol absolu, le réactif de Bradford, le sérum albumine (BSA) qui sont fournis par Biolabo 
S.A. FISMES (France); le dodécyle sulfate sodium (SDS), la malonedialdéhyde (MDA), l'acide thiobarbiturique, le 1,1-diphény-1,2picril hydrazine (DPPH), le 2,4,6-tripyridyl-Striazine (TPTZ), qui sont fournis par SIGMA Chemical (St louis, USA); le chlorure de potassium $(\mathrm{KCl})$ provient de Fisher Bioblock Scientific (France), les solutions étalons de plomb et de cadmium proviennent de Merck KGaA (Durmstalt, Allemagne).

\section{Echantillonnage}

L'étude est conçue pour comparer des personnes d'âge similaire $(\mathrm{P}=0,3732>0,05)$ compris entre 25 et 50 ans avec une moyenne de 34,66 \pm 0,84 ans à Gbodjomé (contrôle) et 35, $04 \pm 0,81$ ans à Kpémé (zone de pollution); ayant des habitudes alimentaires similaires et évoluant dans des circonstances identiques. Ainsi, une enquête à permis d'identifier et de sélectionner deux groupes de 100 personnes, hommes et femmes non fumeurs ayant pour source habituelle de protéines animales des produits de mer pêchés à Gbodjomé pour le groupe de Gbodjomé et à Kpémé pour le groupe de Kpémé.

Environ $10 \mathrm{ml}$ de sang sont prélevés sur chaque sujet après une douche (sans vêtements de travail pour les agents de l'usine), et répartis dans deux tubes de $5 \mathrm{ml}$ garantis sans plomb ni cadmium (bouchons compris) dont l'un avec anticoagulant (EDTA) et l'autre sans anticoagulant et acheminés dans une glacière à la température ambiante au laboratoire d'analyse le même jour. Les prélèvements sont réalisés avec précaution par rapport aux instruments utilisés (aiguilles, tubes, bouchons antiseptiques, gants...). Le sang prélevé sous anticoagulant a servi au dosage du $\mathrm{Cd}$ et $\mathrm{du} \mathrm{Pb}$ et celui prélevé dans des tubes sans anticoagulant pour le dosage des indicateurs du stress oxydatif.

\section{Méthodologie}

Minéralisation des échantillons et détermination des teneurs en $\mathrm{Cd}$ et en $\mathrm{Pb}$

Trois $\mathrm{g}$ de chaque échantillon ajoutés à $2 \mathrm{ml}$ d'acide sulfurique concentré et $1,5 \mathrm{ml}$ d'acide nitrique concentré sont introduits dans des flacons en verre boro silicaté bien fermés avec des capuchons pour éviter d'éventuelles fuites. Les flacons sont ensuite portés au bain marie à $80{ }^{\circ} \mathrm{C}$ pendant 2 heures. Le produit obtenu est dilué avec l'eau distillée jusqu'à 50 $\mathrm{ml}$. La solution ainsi obtenue est utilisée pour la lecture au spectrophotomètre d'absorption atomique à l'électrothermie (marque Varian) couplé à un micro-ordinateur.

\section{Dosage protéique et des bioindicateurs du stress oxydatif}

Les échantillons réservés à cet effet, sont centrifugés à 3000 tours/minute pendant 15 minutes à la température ambiante le même jour. Le plasma est alors utilisé pour déterminer la concentration du MDA et l'activité du FRAP (Ferric Reducing Antioxidant Power). La teneur en protéines totales des échantillons a été estimée par la méthode de Bradford (1976) avec le sérum albumine bovine (BSA) comme standard. La peroxydation des lipides (MDA) a été déterminée en mesurant les substances acides réagissant dans l'acide thiobarbiturique (TBARS) comme décrit par Satoh (1978). L'activité du FRAP a été déterminée en mesurant le potentiel total antioxydant du plasma : $300 \mu \mathrm{l}$ du réactif de FRAP (mélange de $25 \mathrm{ml}$ de tampon acétate ; $2,5 \mathrm{ml} \mathrm{de} \mathrm{F}_{\mathrm{e}}{ }^{3+}$, TPTZ (10 mM.L ${ }^{-1}$ dans 40 mM.L ${ }^{-1}$ de $\mathrm{HCl}$ ) et $2,5 \mathrm{ml}$ de $\mathrm{F}_{\mathrm{e}} \mathrm{Cl}_{3}-6 \mathrm{H}_{2} \mathrm{O}$ ) a été mélangé avec 10 $\mu 1$ de sérum et $30 \mu \mathrm{l}$ d'eau distillée. Le changement de l'absorbance a été mesuré à $593 \mathrm{~nm}$ lorsque le complexe bleu de $\mathrm{F}_{\mathrm{e}}{ }^{2+}$ tripyridyl-5-triazine $\left(\mathrm{F}_{\mathrm{e}}{ }^{2+}-\mathrm{TPTZ}\right) \mathrm{s}$ 'est formé à partir $\mathrm{du} \mathrm{F}_{\mathrm{e}}^{3+}$ oxydé incolore (Nair et al., 2007). Les courbes de calibration sont obtenues à partir d'une solution aqueuse de $\mathrm{F}_{\mathrm{e}} \mathrm{SO}_{4}$ à concentrations variées allant de 10 à $2000 \mu \mathrm{M} . \mathrm{L}^{-1}$. Le réactif actif a été préparé avec $25 \mathrm{ml}$ de tampon acétate ; 2,5 $\mathrm{ml} \mathrm{de} \mathrm{F}_{\mathrm{e}}{ }^{3+}$ TPTZ (10 mM.L ${ }^{-1}$ dans $40 \mathrm{mM} . \mathrm{L}^{-1}$ de $\mathrm{HCl}$ ) et 2,5 $\mathrm{ml} \mathrm{de} \mathrm{F}_{\mathrm{e}} \mathrm{Cl}_{3}-6 \mathrm{H}_{2} \mathrm{O}\left(20 \mathrm{Mm} \cdot \mathrm{L}^{-1}\right)$.

\section{Analyse statistique}

Tous les résultats sont exprimés sous forme de la moyenne \pm SEM et analysés à l'aide du logiciel Graph pad Prism. L'analyse de la variance a été effectuée à l'aide du Test $\mathrm{t}$ 
de Student unpaired. Le seuil de signification est fixé à $\mathrm{P}<0,05$.

\section{RESULTATS}

\section{Cadmie et Plombémie}

Les teneurs en $\mathrm{Cd}$ et en $\mathrm{Pb}$ dans le sang humain à Gbodjomé et à Kpémé sont indiquées dans le Tableau 1. La cadmie et la plombémie à Kpémé (zone de pollution) sont plus élevées que celles de Gbodjomé et plus élevées que les valeurs limites admises par les «Centers for Disease Control and Prevention» et «l'American Academy of Pediatrics » (Marie-Michèle, 2008). D'une manière générale, les teneurs en $\mathrm{Pb}$ sont supérieures à celles du $\mathrm{Cd}$.
Taux de la Malonedialdéhyde (MDA)

Le taux de MDA a significativement augmenté dans le sang des sujets à Kpémé ( $\mathrm{P}$ $<$ 0,0001) par rapport à Gbodjomé. Le pourcentage d'augmentation de la peroxydation des lipides est de $63,46 \%$ (Figure 1).

\section{Potentiel total antioxydant du plasma}

Les valeurs de FRAP ont significativement diminuée dans le sang des sujets à Kpémé $(\mathrm{P}<0,0001)$ par rapport à Gbodjomé. Le pourcentage de diminution du potentiel total antioxydant du plasma sanguin est de $65,85 \%$ (Figure 2).

Tableau 1: Cadmie et Plombémie à Gbodjomé et à Kpémé.

\begin{tabular}{lcc}
\hline Localités & Cadmie $(\boldsymbol{\mu g} / \mathbf{K g})$ & Plombémie $(\boldsymbol{\mu g} / \mathbf{K g})$ \\
\hline Gbodjomé & $1,01 \pm 0,11$ & $87,27 \pm 12,57$ \\
Kpémé & $13,53 \pm 1,07^{* * *}$ & $384,09 \pm 22,45 * * *$ \\
$\%$ de différence & 1239 & 340 \\
\hline \multicolumn{2}{c}{ Les résultats sont exprimés sous forme de moyenne \pm ESM de 100 échantillons. Significativement } \\
différent : $* * * \mathrm{P}<0,0001$.
\end{tabular}

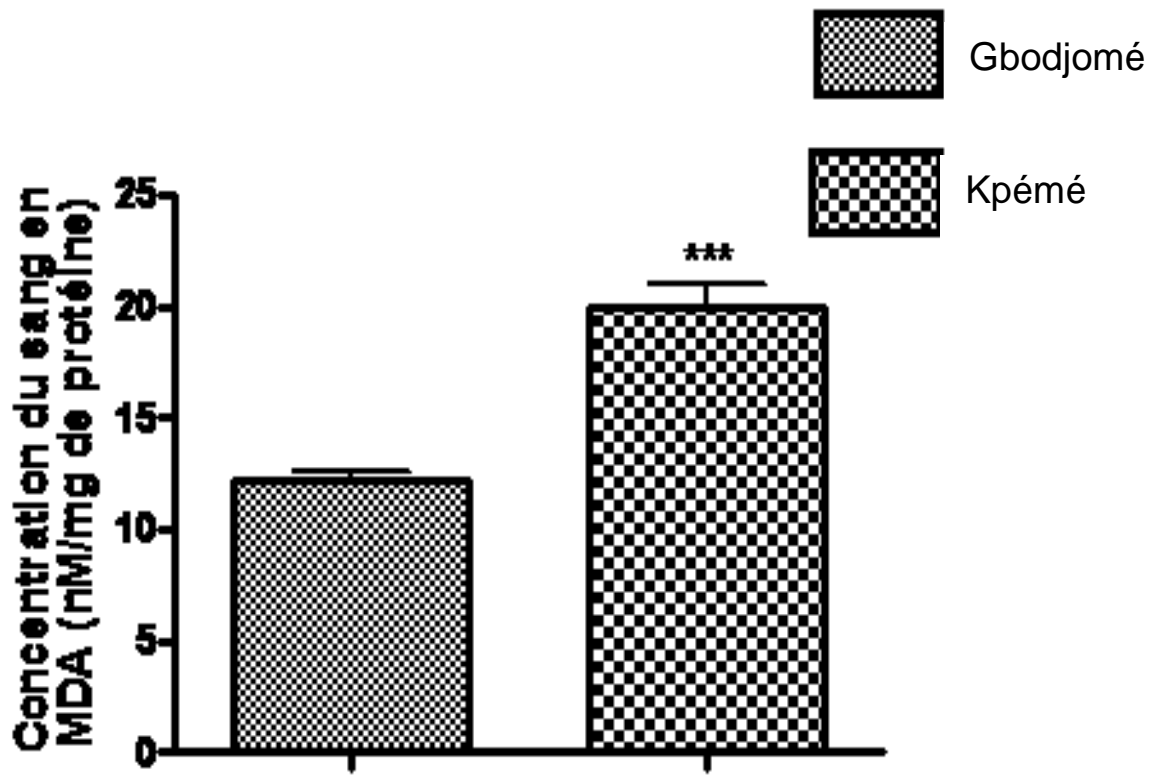

Figure 1: Taux de la malonedialdéhyde (MDA) dans le sang humain selon les localités. Les valeurs sont exprimées sous forme de moyenne \pm ESM de 100 échantillons. Significativement différent par rapport au contrôle : *** $\mathrm{P}<0,0001$. 


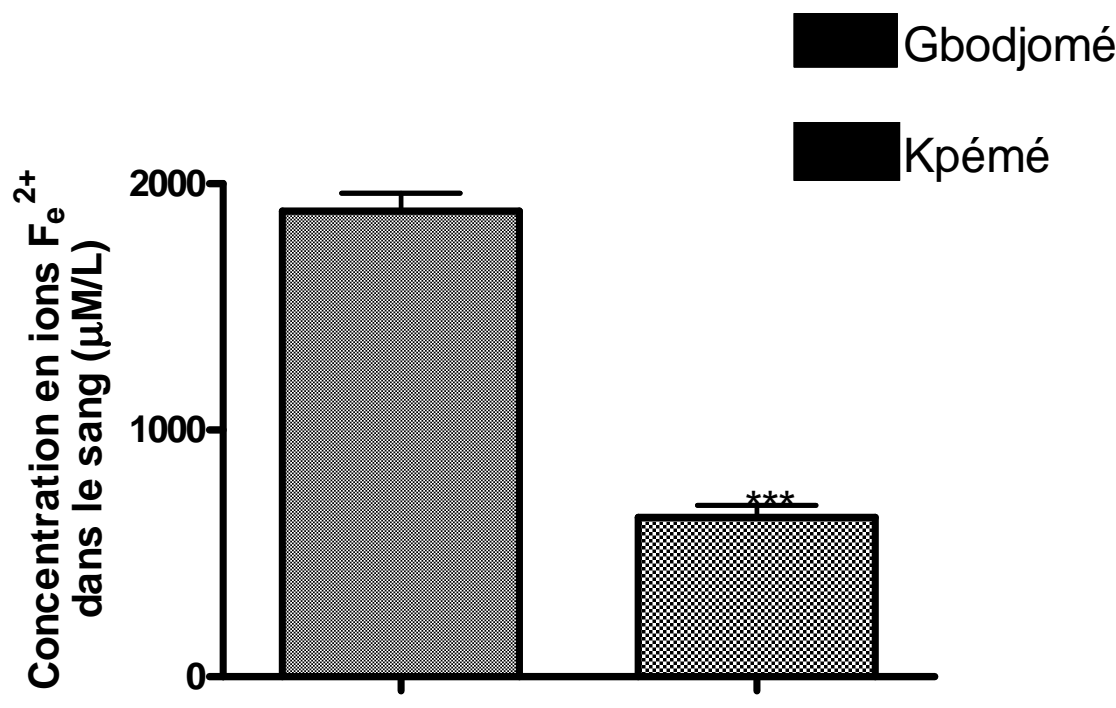

Figure 2: Concentration en ions $\mathrm{F}_{\mathrm{e}}{ }^{2+}$ (valeur du FRAP) dans le sang humain selon les localités. Les valeurs sont exprimées sous forme de moyenne \pm ESM de 100 échantillons. Significativement différent par rapport au contrôle : *** $\mathrm{P}<0,0001$.

\section{DISCUSSION}

L'industrie est l'une des principales sources de contamination des métaux lourds toxiques tels que le $\mathrm{Cd}$ et le $\mathrm{Pb}$ (Farombi et al., 2007 ; Yildirim et al., 2011 ; Mélila et al., 2012). Au Togo, la côte marine est polluée du fait, entre autres des activités industrielles et des études sont menées concernant cette pollution notamment celle due aux activités industrielles de la SNPT qui traite le phosphate togolais (Gnandi, 1998 ; Bodjock, 2003 ; Tchangbédji et al., 2003 ; Abbe, 2004 ; NSE/ONUDI, 2007 ; Awadé, 2010) et son impact sur la biodiversité marine (Abbe, 2004 ; Mélila et al., 2012). Cependant, l'évaluation des différentes concentrations des éléments traces métalliques tels que le $\mathrm{Cd}$ et le $\mathrm{Pb}$, dans le sang humain n'a pas encore été faite et il n'existe aucune donnée chez l'homme, sur les biomarqueurs du stress oxydatif, bioindicateurs de la pollution environnementale (Farombi et al., 2007) et conséquence de la bioaccumulation des métaux lourds toxiques (Yildirim et al., 2011) dans les zones faisant l'objet de cette pollution, notamment à Kpémé. Dans cette étude, nous avons évalué les teneurs du Cd et $\mathrm{du} \mathrm{Pb}$, principaux métaux lourds toxiques contenus dans les boues, déchets et poussières du phosphate togolais et bioaccumulés par l'homme par voies trophique, respiratoire et cutanée, de même que le taux de certains biomarqueurs du stress oxydatif dans le sang des individus exposés.

Nos résultats indiquent que la Cadmie et la Plombémie sont élevées chez des sujets habitant à Kpémé, par rapport à Gbodjomé ; ce qui a induit une peroxydation lipidique de même qu'une diminution du potentiel total antioxydant du plasma sanguin.

La concentration très élevée des métaux dans le sang à Kpémé peut s'expliquer par le fait que l'usine de la SNPT qui s'y trouve, induit plusieurs sources de contamination des métaux lourds à l'homme. L'homme est contaminé à partir de la consommation des produits de la mer qui bioaccumulent ces métaux à partir des boues et déchets rejetés dans la mer (Abbé, 2004) et à partir des produits maraîchers et agricoles 
cultivés à proximité de l'usine qui bioaccumulent les éléments traces métalliques à partir des poussières et gaz rejetés par l'usine dans l'atmosphère (Aduayi-Akue, 2010). En effet, les travaux antérieurs (Abbé, 2004 ; Aduayi-Akue, 2010) ont montré que les concentrations des métaux dans les produits de mer, légumes et produits agricoles au niveau de Kpémé sont supérieures aux normes admises pour une consommation (WHO, 1992), indiquant une contamination des poissons et produits agricoles aux métaux lourds et en tant que tel par la chaîne alimentaire, les consommateurs peuvent êtres vulnérables à la toxicité de ces métaux. La forte bioaccumulation du $\mathrm{Cd}$ et du $\mathrm{Pb}$ par l'homme serait aussi due à la respiration et au contact dans une atmosphère polluée par les poussières et gaz rejetés à la suite du traitement du minerai de phosphate togolais et contenant ces métaux (NSE/ONUDI, 2007). Les concentrations du sang en $\mathrm{Cd}$ et en $\mathrm{Pb}$ à Kpémé sont très supérieures à celles retrouvées dans les produits de mer par Abbé (2004), dans les produits agricoles par Aduayi-Akue (2010) et dans les poussières par Awadé (2010); ce ci indique une disponibilité de l'organisme à la boaccumulation des éléments traces métalliques comme le $\mathrm{Cd}$ et le $\mathrm{Pb}$. Cela peut s'expliquer aussi par le caractère ubiquiste des métaux et leur faible élimination par l'organisme. Dans notre étude, la plombémie très élevée par rapport à la cadmie s'expliquerait par le fait que les aliments consommés concentrent plus le $\mathrm{Pb}$ que le $\mathrm{Cd}$ comme l'ont montré les études antérieures (Abbé, 2004 ; Aduayi-Akue, 2010). Cette bioaccumulation du $\mathrm{Cd}$ et $\mathrm{du} \mathrm{Pb}$ à partir de la consommation des aliments contaminés à des concentrations supérieures aux seuils limites autorisés par l'Organisation Mondiale pour la Santé (OMS) pour une utilisation comme aliment peut altérer intensément la santé de l'homme (Miquel, 2001; OMS, 2004 ; Gnandi et al., 2008). En effet, le $\mathrm{Cd}$ et le $\mathrm{Pb}$ sont des éléments toxiques, après le mercure qui touchent les organes cibles tels que les reins, le foie, le système nerveux, le sang et peuvent être source de maladies cardiovasculaires (OMS, 2004). Par ailleurs, la présence des métaux lourds toxiques dans les organismes est source de radicaux libres provoquant le stress oxydatif (Farombi et al., 2007 ; Yildirim et al., 2011; Mélila et al., 2012). Nos résultats ont montré une élévation significative de la peroxydation lipidique dans le sang au niveau du site pollué (Kpémé) par rapport à la zone de référence (Gbodjomé). Cette augmentation peut être attribuée à l'accumulation du $\mathrm{Cd}$ et du $\mathrm{Pb}$ dans le sang tel que l'indiquent nos données. En effet, l'accumulation de ces métaux catalyse les ROS capables d'endommager les molécules comme l'ADN, les protéines et les lipides (Pandey et al., 2003; Farombi et al., 2007 ; Yildirim et al., 2011). L'augmentation du taux sanguin de la MDA observée à Kpémé par rapport à Gbodjomé montre une lutte de l'organisme contre le stress oxydatif accru induit par les métaux lourds. Cette condition pathologique est confirmée par la baisse du potentiel total antioxydant du plasma sanguin, exprimée par une faible concentration du sang en ions $\mathrm{F}_{\mathrm{e}}{ }^{2+}$ à Kpémé par rapport à Gbodjomé. En effet, les valeurs de FRAP (Ferric Reducing Antioxidant Power) baissent significativement dans le cas d'un stress oxydatif accru. Ces résultats sont conformes à ceux de Agbonon et Gbéassor (2009) qui ont observé une baisse sensible du potentiel total antioxydant du plasma chez les rats wistar intoxiqués par le $\mathrm{CCl}_{4}$, chez lesquels la peroxydation lipidique avait notamment augmentée.

\section{Conclusion}

Les travaux de recherche antérieurs ont montré que l'environnement de la côte togolaise est pollué par les déchets de l'usine de la SNPT et des travaux récents indiquent une bioaccumulation des métaux lourds toxiques par les produits de la mer à partir des effluents qui y sont rejetés (Abbé, 2004) et par les produits agricoles cultivés à proximité de cette usine à travers les gaz et poussières rejetés dans l'atmosphère (Aduayi-Akue, 2010). Cette situation rend vulnérable 
l'homme qui est en bout de la chaîne trophique par rapport aux métaux lourds toxiques contenus dans les aliments consommés. La présente étude montre que l'organisme humain présente une disponibilité à la bioaccumulation des éléments traces métalliques. Les fortes concentrations du $\mathrm{Cd}$ et $\mathrm{du} \mathrm{Pb}$ dans le sang des sujets à Kpémé par rapport à Gbodjomé sont dues d'une part à une consommation d'aliments contaminés à partir des déchets rejetés dans la mer et dans l'atmosphère et d'autre part, à une contamination directe par voie respiratoire et par voie d'absorption cutanée à cause des activités industrielles de la SNPT. Cette bioaccumulation du $\mathrm{Cd}$ et $\mathrm{du} \mathrm{Pb}$ par les sujets exposés serait la cause de la péroxydation lipidique accrue et de la diminution significative du potentiel total antioxydant du plasma sanguin observées à kpémé, indiquant l'induction d'un stress oxydant chez les individus exposés. Cette étude devrait s'étendre aux localités voisines de Kpémé touchées par la pollution induite dans la zone par l'usine de traitement du minerai de phosphate togolais afin de comparer les impacts par rapport à la position de chaque localité.

\section{REFERENCES}

Abbé KD. 2004. Evaluation des conséquences du rejet des déchets phosphatés dans la mer : étude de la bio-accumulation de quelques éléments chimiques toxiques par les espèces animales. Cas du plomb et du cadmium. Mémoire d'ingénieur de Travaux en GEE/ESTBA, Université de Lomé, Togo, p. 6 ; 21 ; 25 ;29-30.

Aduayi-Akue AA. 2010. Evaluation de la pollution par les métaux lourds des sols et des produits agricoles autour des sites de traitement des phosphates de Kpémé (Sud Togo) : cas du cadmium, du plomb, du nickel et du cuivre. Mémoire de fin de formation en DEA de Biologie de Développement, option Gestion de l'Environnement, Faculté Des Sciences, Université de Lomé, Togo, p. 51-69.
Aït-Aïssa S, Palluel O, Porcher JM. 2003. Biomarqueurs précoces d'écotoxicité. Rapport final BCRD, Ministère de l'Ecologie du Développement Durable, INERIS, Paris.

Agbonon A, Gbéassor M. 2009. Hepatoprotective effect of Lonchocarpus sericeus Leaves in $\mathrm{CCl}_{4}$ induced liver damage. Journal of Herbs, Spices and Medical Plants, 15: 216-226.

Alméida JA, Diniz YS, Marques SFG, Faine LA, Ribas BO, Burneiko RC, Novelli ELB. 2002. The use of the oxydative stress response as biomarkers in Nile tilapia (Oreochromis niloticus) exposed to in vivo cadmium contamination. Environment International, 27: 673-679.

Awade M. 2010. Les impacts environnementaux et socioéconomiques de l'exploitation du gisement de phosphate de Hahotoé-Kpogamé. Mémoire de Maîtrise ès Lettre et Sciences Humaines, Option Géomorphologie et Gestion des Milieux Naturels, p. 80-86.

Bodjock K. 2003. Etude chimique de l'impact sur l'environnement du rejet dans la mer des effluents du traitement des phosphates de Hahotoé-Kpogamé (Togo). Mémoire d'Ingénieur de Travaux en GEE/ESTBA, Université de Lomé, Togo, P. 6-30.

Bradford M. 1976. A rapid and sensitive method for the quantitation of microgram quantities of proteins utilizing the principle of protein dye-binding. Anal. Biochem., 72: 248-254.

Charissou AM, Cossu-Leguille C, Vasseur P. 2004. Relationship between two oxidative stress biomarkers, malondialdehyde and 8-oxo-7,8-dihydro-2'-deoxyguanosine, in the freshwater bivalve Unio tumidus. Sci. Total Environment, 322: 109-122.

Farombi EO, Adelowo OA, Ajimoko YR. 2007. Biomarkers of oxidative stress and heavy metal levels as indicators of environmental pollution in African Cat Fish (Clarias gariepinus) from Nigeria Ogun River. International Journal of 
Environmental Research and Public Health, 4(2): 158-165.

Favier A. 2003. Le stress oxidant: intérêt conceptuel et expérimental dans la compréhension des mécanismes des maladies et potentiel thérapeutique. Mécanismes biochimiques. L'Actualité Chimique, 18: 108-113.

Gnandi K. 1998. Cadmium et autres polluants inorganiques dans les sols et les sédiments de région côtière du Togo : étude géochimique. Thèse de l'Université Friedrich Alexandre d'Erlangen Nuremberg, Republique fédérale d'Allemagne, p. 10-11; 137.

Gnandi K, Tozo K, Edorh AP, Abi H, Agbeko K, Amouzouvi K, Gnon B, Gado T, Kili K, Bouchet P, Akpagana K. 2008. Bioaccumulation de certains elements métalliques dans les produits maraîchers cultivés sur les sols urbains le long de l'autoroute Lomé-Aného, Sud Togo. Acta Bot. Gallica, 115(3): 415-426.

Halliwell B, Gutteridge J. 1999. Free Radicals in Biology and Medicine ( $3^{\text {rd }}$ edn). Oxford University Press: Oxford; 617-783.

Legault J. 2007. Les propriétés antioxydantes du Bleuet. European Journal of Clinical Nutrition, 61: 431-433.

Marie-Michèle M. 2008. Center of diseases contrôl and prevention, National repport on human exposure to environnemental chemicals CDC.

Mélila M, Poutouli W, Amouzou KS, Tchangbédji G, Thaou M, Doh A. 2012. Évaluation de l'impact du rejet des déchets phosphates dans la mer sur la biodiversité marine dans trois localités côtières au Togo à partir des biomarqueurs du stress oxydatif chez Sphyraena barracuda (HECKEL, 1843). Int. J. Biol. Chem. Sci., 6(2): 820-831.

Miquel G. 2001. Effet des métaux lourds sur l'environnement et la santé, rapport 261 ; Office Parlementaire d'Evaluation des Choix Scientifiques et Technologiques, p.365.
Nair V-P, Dairam A, Agbonon A, Arnason BC, Foster, Kanfer I. 2007. Investigation of the antioxidant activity of African potato (Hypoxis bemerocallidea). J. Agric. Food Chem., 55(5): 1707-1711.

Nordberg J, Arner ESJ. 2001. Reactive oxygen species, antioxidants and the mammalian thiredoxin system. Journal of Free Radicals in Biology and Medicine, 31(11): 1287-1312.

NSE/ONUDI. 2007. Réduction des déchets des mines de phosphate dans le GCLME. Projet de démonstration du Togo, Rapport final, P. 78-83.

OMS. 2004. Guidelines for Food and Drink Water Quality, Chemical Fact Sheets $\left(3^{\text {rd }}\right.$ edn). OMS: Geneva, Swiss; 460p.

Pandey S, Parvez S, Sayeed I, Haque R, BinHafeez B, Raisuddin S. 2003. Biomarkers of oxidative stress: a comparative study of river Yamuna Fish Wallago attu (BI. and Schn.). Sci. Total Environ., 309: 105115.

Satoh K. 1978. Serum lipid peroxide in cerebrovascular disorders determined by a new colorimetric method. Cl. Chim. A., 901: 37-43.

Servais S. 2004. Altération mitochondriale et stress oxydant pulmonaire en réponse à l'ozone: effet de l'âge et d'une suplémentation en oméga-3. Thèse de Doctorat de 1'Université Claude BernardLyon1, p. 17-40.

Tchangbedji G, Djeteli G, Kili A, Savariault MJ, Lacoutl J. 2003. Chimical and structural characterization of natural phosphate of Hahotoé (Togo). Bul. Chim., Ethiop., 17(2): 1-8.

WHO. 1992. Inorganic Lead, Environmental Health Criteria (vol. 165). WHO: Geneva.

Yildirim NC, Benzer F, Danabas D. 2011. Evaluation of environmental pollution at Munzur river of Tunceli applying oxidative stress biomarkers in Cappota trutta (Heckel, 1843). J. Anm. Plant Sci., 21(1): 66-71. 